\title{
Evidence for crater ejecta on Venus tessera terrain from Earth-based radar images
}

\author{
Bruce A. Campbell ${ }^{\mathrm{a}, *}$, Donald B. Campbell ${ }^{\mathrm{b}}$, Gareth A. Morgan ${ }^{\mathrm{a}}$, Lynn M. Carter ${ }^{\mathrm{c}}$, Michael C. Nolan ${ }^{\mathrm{d}}$, \\ John F. Chandler ${ }^{\mathrm{e}}$ \\ a Smithsonian Institution, MRC 315, PO Box 37012, Washington, DC 20013-7012, United States \\ ${ }^{\mathrm{b}}$ Cornell University, Department of Astronomy, Ithaca, NY 14853-6801, United States \\ ' NASA Goddard Space Flight Center, Mail Code 698, Greenbelt, MD 20771, United States \\ ${ }^{\mathrm{d}}$ Arecibo Observatory, HC3 Box 53995, Arecibo 00612, Puerto Rico \\ e Smithsonian Astrophysical Observatory, MS-63, 60 Garden St., Cambridge, MA 02138, United States
}

\section{A R T I C L E I N F O}

\section{Article history:}

Received 12 June 2014

Revised 14 November 2014

Accepted 24 November 2014

Available online 5 December 2014

\section{Keywords:}

Venus, surface

Radar observations

Cratering

\begin{abstract}
A B S T R A C T
We combine Earth-based radar maps of Venus from the 1988 and 2012 inferior conjunctions, which had similar viewing geometries. Processing of both datasets with better image focusing and co-registration techniques, and summing over multiple looks, yields maps with 1-2 km spatial resolution and improved signal to noise ratio, especially in the weaker same-sense circular (SC) polarization. The SC maps are unique to Earth-based observations, and offer a different view of surface properties from orbital mapping using same-sense linear ( $\mathrm{HH}$ or VV) polarization. Highland or tessera terrains on Venus, which may retain a record of crustal differentiation and processes occurring prior to the loss of water, are of great interest for future spacecraft landings. The Earth-based radar images reveal multiple examples of tessera mantling by impact "parabolas" or "haloes", and can extend mapping of locally thick material from Magellan data by revealing thinner deposits over much larger areas. Of particular interest is an ejecta deposit from Stuart crater that we infer to mantle much of eastern Alpha Regio. Some radar-dark tessera occurrences may indicate sediments that are trapped for longer periods than in the plains. We suggest that such radar information is important for interpretation of orbital infrared data and selection of future tessera landing sites.
\end{abstract}

Published by Elsevier Inc.

\section{Introduction}

Much of the surface of Venus is characterized by volcanic plains and flows from sources that include dome fields, coronae, and large shields. Any record of geologic processes that occurred prior to the emplacement of the plains, and especially evidence of interactions with water, is likely confined to the highly deformed upland or tessera deposits. Tessera terrain comprises $7-8 \%$ of the total surface area, occurs preferentially on and near crustal plateaus, and might represent a widespread basement beneath the plains (Barsukov et al., 1986; Ivanov and Head, 1996; Ivanov and Head, 2011; Hansen et al., 1999). Felsic mineralogy in these uplands suggesting differentiation in the presence of water, similar to Earth's continents, has been inferred from flyby and orbital infrared observations (Helbert et al., 2008; Hashimoto et al., 2008; Basilevsky et al., 2012). Definitive answers regarding tessera composition will

\footnotetext{
* Corresponding author.

E-mail address: campbellb@si.edu (B.A. Campbell).
}

require more detailed remote sensing or a landed mission (Basilevsky et al., 2007; Report of the Venus Science and Technology Definition Team, 2009). Our knowledge, however, of weathering and other physical processes operating in the tesserae (Campbell et al., 1999; Arvidson et al., 1994; Bindschadler and Head, 1988, 1989), and how the visible surface has been modified by crater ejecta (Campbell et al., 1992; Garvin, 1990), remains very limited. The latter topic is of particular importance given the unique nature of distal impact crater deposits on Venus.

The distribution of impact debris on Venus has many similarities with crater deposits on other planetary bodies, but the dense atmosphere affects the formation and distribution of fine-grained distal ejecta. The orbital Magellan radar-mapping mission revealed radar-dark "parabolas" associated with numerous impact craters, and geologic studies (Campbell et al., 1992; Herrick and Phillips, 1994, McKinnon et al., 1997) attributed them to an interaction between fine material ejected from the crater and the dense atmosphere. Larger craters create an ejecta curtain whose top escapes the atmosphere, allowing particles to travel up to $2000 \mathrm{~km}$ on 
the prevailing winds as they settle back to the surface to form a parabolic-shaped deposit opening to the west (Vervack and Melosh, 1992; Schaller and Melosh, 1998). These deposits mantle and subdue surface roughness at the few-cm scale, reducing the radar backscatter cross section.

Parabolic ejecta signatures are evident for 60 or more craters on the plains of Venus, and many more craters have radar-dark "halo" deposits concentric to the rugged, radar-bright rim material. Only in a few instances, such as at Magnani crater in Danu Montes or Wilma crater in Mangan-Gurme Tessera, are extended deposits noted to cross tesserae or densely ridged terrain (Campbell et al., 1992; Carter et al., 2004). Current parabolas and haloes manifest a range of states, varying from homogeneous radar-dark signatures to apparent thinning, exhumation of buried features, and development of streaks that parallel the east-west wind pattern at the surface. Based on this evidence for erosion, and the absence of radar-dark parabolas or haloes around other craters, several authors have suggested that they represent a coarse guide to crater age, with total removal occurring over timescales of perhaps $35 \pm 15$ m.y. (Izenberg et al., 1994; Schaller and Melosh, 1998; Bondarenko and Head, 2009).

It remains uncertain whether every crater of a given diameter produces a nearly identical parabola and halo, or if there are significant bolide-related, atmospheric, entry angle, or target effects on ejecta distribution. In addition, Magellan mapping utilized a radar polarization that may not reveal the full extent of fine-grained crater deposits, especially in rough terrain. We thus cannot readily trace impact deposits into the tesserae in Magellan images, nor can we assert, for a particular crater, that the absence of parabolic signatures in the plains guarantees a paucity of ejecta in nearby highlands. Basilevsky et al. (2004) present a map of Venus which includes estimated parabolic ejecta patterns from all craters $>11 \mathrm{~km}$ in diameter, regardless of their current associated radar signatures. This mapping suggests that $80-90 \%$ of the surface has been affected by debris from at least one impact crater, and the authors note the importance of this finding to interpretation of existing in-situ analyses and selection of future landing sites.

New studies are required to better understand the relationship of distal ejecta with other possible indicators of crater age, and to search for patches of fine-grained debris emplaced in the tesserae as a guide to remote sensing interpretation and landing site selection. In this work, we address these issues using Earth-based radar mapping of Venus from the 1988 and 2012 inferior conjunctions. Section 2 discusses the observations, data reduction, and how the dual-polarization echoes offer significant new information on surface properties relative to single-polarization mapping, using a case study of two relatively young craters. In Section 3 we study three tessera regions that show evidence for mantling deposits related to impact craters. Section 4 summarizes our results and discusses implications for future exploration.

\section{Polarimetric radar observations}

The radar maps presented here are obtained using the Arecibo Observatory $12.6-\mathrm{cm}(2380 \mathrm{MHz})$ transmitter. Data were collected in 1988 with Arecibo alone (Campbell et al., 1989), and in 2012 with Arecibo transmitting and the Green Bank Telescope receiving the echoes. The Earth-based data use a circularly polarized transmitted signal, and measure echoes in two modes termed "opposite-circular" or OC and "same-circular" or SC. Delay resolution was $4 \mu$ s in 1988 and $3.8 \mu$ s in 2012, yielding spatial resolution of $1-2 \mathrm{~km}$ along the range direction. Each $270 \mathrm{~s}$ of coherent integration time yields one "look", with 1-2 km spatial resolution, from Doppler processing. We converted the delay-Doppler images to latitude-longitude format, at $1-\mathrm{km}$ posting, using information on the sub-radar point location, limb-to-limb bandwidth, and apparent spin axis orientation at the time of each look (e.g. Campbell et al., 2007).

The 1988 data were re-processed to compensate for small errors in the Doppler shift estimate, based on calculated ephemerides, which led to modest smearing of the images. To make the image products used in our analysis, we averaged looks for the northern (61 total over the 1988 and 2012 datasets) and southern (34 total from 2012 alone) hemisphere, using image correlation techniques to compensate for minor offsets in the mapping process. Mapping is limited to the hemisphere of Venus that faces Earth at inferior conjunction (Fig. 1). The sub-earth latitude and longitude were similar between the 1988 and 2012 observations, since the orbital geometry for the two planets repeats closely every eight years.

Earth-based observations cannot resolve echoes from the northern and southern hemispheres that arrive with identical time delay and Doppler shift. This ambiguity is mitigated in part by pointing the transmitting telescope north or south of the planet to reduce reflections from the undesired hemisphere. The unwanted reflections are further reduced by averaging data collected over a period of time as the apparent spin axis changes in orientation. The final maps, especially in the OC polarization, have residual blurred features due to radar-bright landforms in the opposite hemisphere.

The polarization characteristics of a radar echo strongly influence the interpretation of surface properties (e.g., Campbell and Campbell, 1992; Carter et al., 2004, 2006). Like-polarized linear (HH or VV) or opposite-sense circular (OC) signals are strongly affected by the tilt of the surface on few-meter to hundreds of meters horizontal scales; radar-facing slopes appear very bright, while away-facing slopes are much darker (e.g., Hagfors, 1964). Magellan used either $\mathrm{HH}$ or VV polarization, so images of the tesserae are dominated by strong radar "glints" created by the large-scale slopes of their ridge and valley structure. In contrast, SC reflections, similar to cross-polarized linear echoes (HV or $\mathrm{VH}$ ), are strongly modulated by diffuse scattering from the $\mathrm{cm}$ - to decimeter-scale roughness of the surface, and large-scale

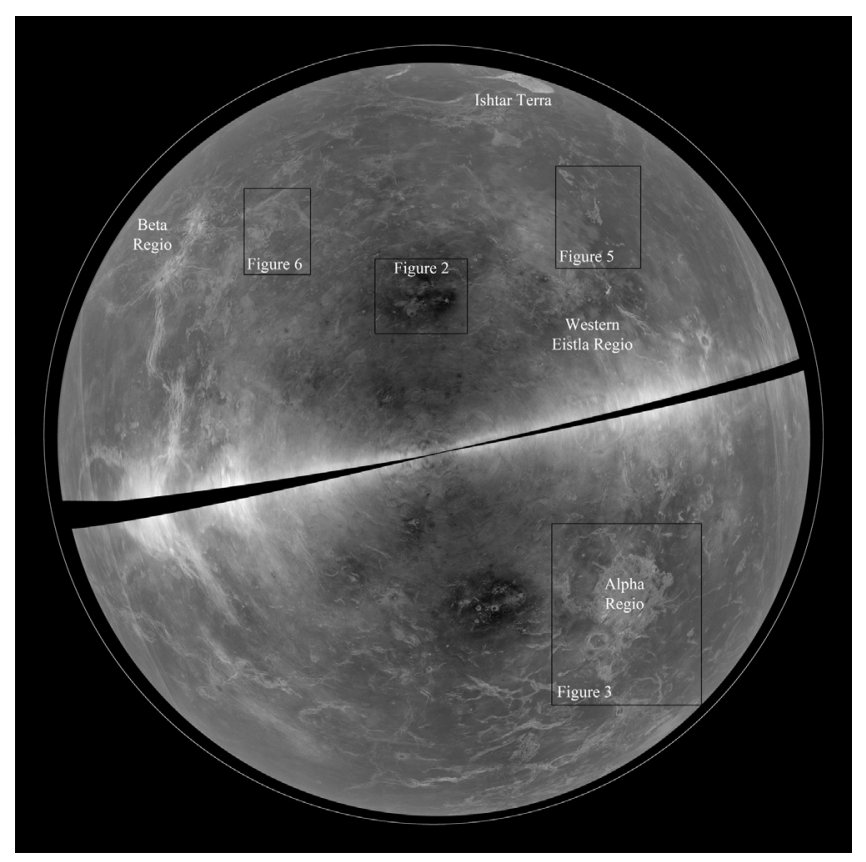

Fig. 1. Earth-based opposite-circular $(O C)$ polarization radar images of Venus collected in 2012 . Orthographic map projection centered on $0^{\circ} \mathrm{N}, 330^{\circ} \mathrm{E}$. The black diagonal band at center represents areas too close to the Doppler "equator" to obtain well-resolved image data. Approximate regions of coverage in major subsequent figures are shown in black outlines. 
slopes have much less effect (e.g., Campbell and Shepard, 1996). This means that the SC echoes will exhibit a more obvious decline due to smoothing of the surface by fine-grained debris, especially in more rugged "background" terrain, than observed in the $\mathrm{HH}$, $\mathrm{VV}$, or OC modes. Such behavior is noted in lunar radar studies of rock-poor crater deposits (Ghent et al., 2005) and fine-grained pyroclastic ash (Campbell et al., 2008).

These polarimetric backscatter behaviors are well illustrated in images of the craters Aurelia (31 km diameter) and Seymour (63 km diameter). In a Magellan 12.6-cm wavelength image, using $\mathrm{HH}$ polarization, the floor and near-rim ejecta of Aurelia are radarbright, consistent with rugged terrain at a range of scales from a few $\mathrm{cm}$ to tens of meters (Fig. 2a). The nearby crater Seymour has a radar-dark floor indicating post-impact lava flooding, and a large northeast-trending complex of radar-bright flows formed by ejection of melt from the crater cavity (Chadwick and Schaber, 1993). Aurelia has a small radar-dark halo around its near-rim ejecta, and a clear parabolic ejecta deposit that crosses the impact-melt complex of Seymour (Campbell et al., 1992). The lower $12.6-\mathrm{cm}$ radar echo from the parabola is attributed to a fine-grained ( $\ll 1 \mathrm{~cm}$ diameter) deposit (Vervack and Melosh, 1992) with minimal surface roughness at the few-cm spatial scale (Campbell et al., 1992). Seymour has patches of a near-rim dark halo but no evidence of a parabola, so current models would suggest that the crater is at least as old as the parabola retention age of about $35 \pm 15$ m.y. (Schaller and Melosh, 1998).
The OC polarization Earth-based image shows a similar spatial extent for the Aurelia radar-dark parabolic deposits, but does indicate a somewhat different pattern of low returns within these bounds (Fig. 2b). This may arise due to differences in the incidence angle of the two observations (about $45^{\circ}$ for Magellan and $20-25^{\circ}$ for the Earth-based map), which affects the penetration depth of the signal in the fine-grained mantling material (Carter et al., 2004). Interestingly, the SC polarization image shows a greater degree of echo attenuation over the impact melt flows of Seymour (Fig. 2c), revealing significant and contiguous debris mantling more clearly than the $\mathrm{HH}$ or OC images. We propose that in the rougher melt-sheet terrain, the $\mathrm{HH}$ and $\mathrm{OC}$ echoes are enhanced by radar-facing "facets" created by local slopes on the few-meter and larger scale, mitigating the loss of signal strength created by the mantling material. The SC reflections have little sensitivity to such facet structures, so the effect of the fine-grained debris in smoothing small-scale roughness is more evident. This case study motivated our search for fine crater ejecta in the tesserae based on the 1988 and 2012 SC images.

\section{Evidence for crater ejecta in the tessera}

In this section, we first review the current inventory of craterrelated parabolas on Venus, and fit empirical curves to their dimensional parameters. We then study three tessera regions that
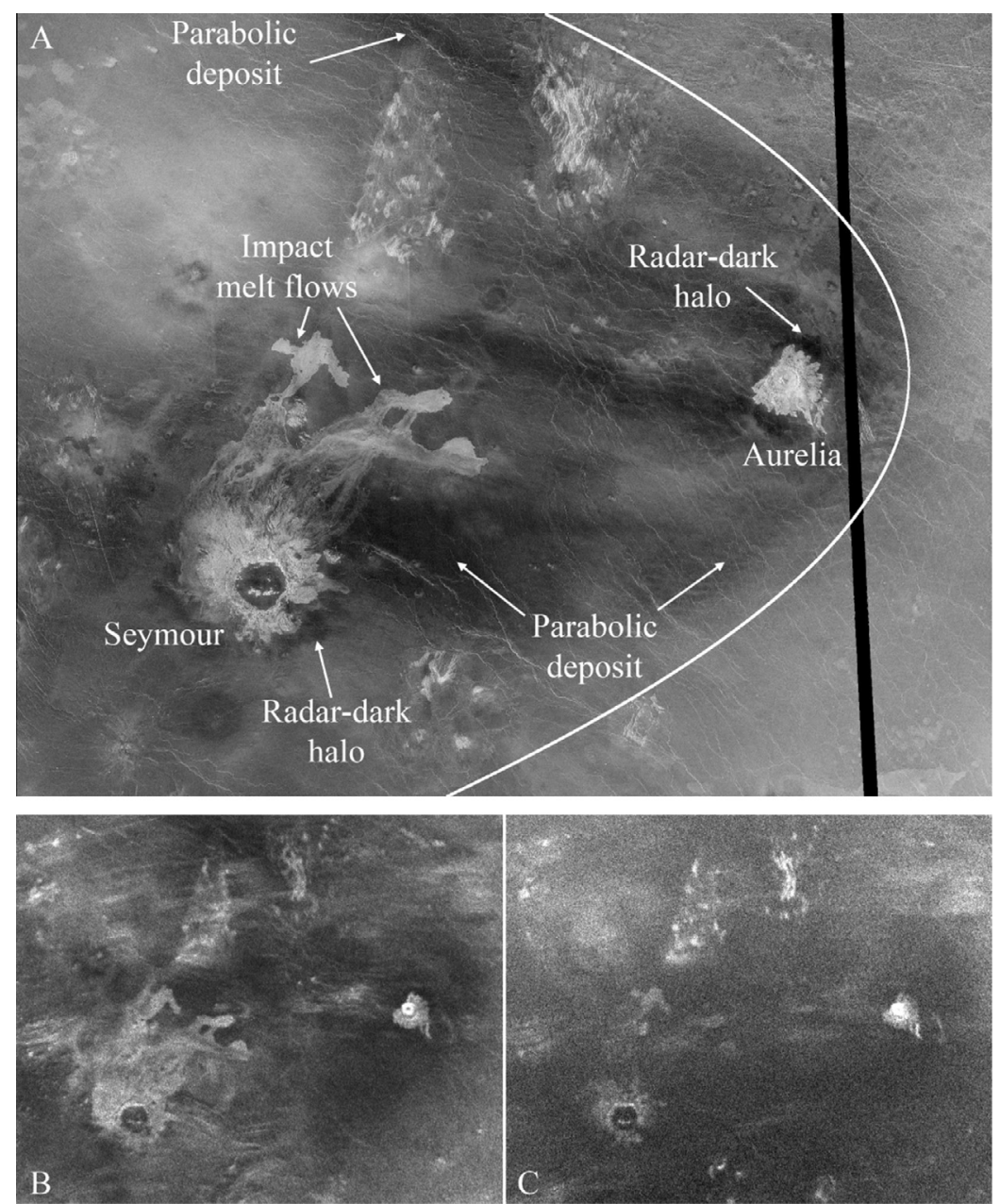

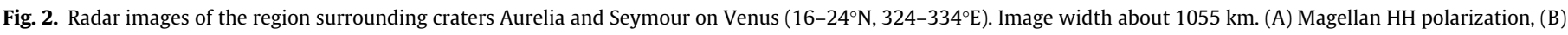

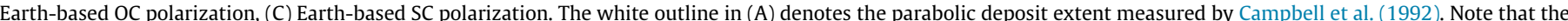

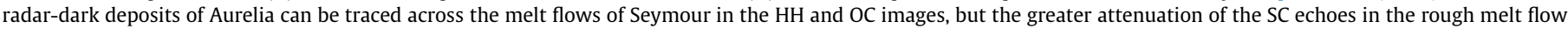
terrain reveals a more contiguous regional mantling. 
exhibit reduced radar echo strength, especially in the SC polarization, relative to some regional average level. For each study, we compare the spatial pattern of lower SC radar echo strength to the predictions of the dimensional models and the expected aeolian degradation of parabolas, and discuss the implications for the presence of fine-grained ejecta in the highlands.

\subsection{Parabola dimensions}

The distribution of about 60 parabolic ejecta deposits across the plains regions of Venus was documented based on Magellan data, with tabulated values for the eastward offset of the parabola vertex from the crater and the maximum width and westward range of the deposit (Schaller and Melosh, 1998; Campbell et al., 1992; Basilevsky et al., 2004). We made simple fits of these parameters as functions of the crater radius, $r$. Eastward offset of the parabola vertex from the crater center (in $\mathrm{km}$ ) is fit by $L_{\mathrm{c}}=10.52 \mathrm{r}$. Maximum range westward from the vertex (in $\mathrm{km}$ ) is given by $L_{\mathrm{T}}=432+$ $25.55 r$. The half-width of the parabola (in $\mathrm{km}$ ) from a centerline with westward distance from the vertex, $L$, is $(12.78+0.47 r) L^{1 / 2}$. Schaller and Melosh (1998) and Campbell et al. (1992) did not map the parabolas into the tesserae, so in some locales the measured range and width will represent minimum values.

\subsection{Stuart crater and Alpha Regio}

We first examine the region near Stuart crater $(69 \mathrm{~km}$ diameter), which has a parabolic ejecta pattern in the surrounding plains as seen by Magellan (Fig. 3a). To the west of Stuart lies Alpha Regio, a $1300-\mathrm{km}$ wide crustal plateau comprising tessera terrain (Bindschadler et al., 1992; Bender et al., 2000; Gilmore and Head, 2000). In the Magellan image (Fig. 3a), the dense network of tessera-forming ridges and troughs is evident across Alpha Regio, as are small, radar-dark patches attributed to smooth-surfaced volcanic flows. Subtle differences in overall radar brightness across Alpha do occur, correlated with modest changes in microwave emissivity (Pettengill et al., 1992). The Earth-based SC radar image reveals a more dramatic variation in backscatter strength, with a generally lower $(-1.2$ to $-1.4 \mathrm{~dB}$, or about $25 \%)$ average in the eastern-central portion of the plateau indicated by the orange outline in Fig. 3.

We propose that the low SC radar echo is due to mantling of the tessera terrain by fine-grained debris of the Stuart parabola. The orange outline in Fig. 3 implies an 1800-2000 km maximum distance from the parabola vertex for these deposits, considerably larger than the $1220 \mathrm{~km}$ noted by Schaller and Melosh (1998) and plotted in white on Fig. 3. The lower SC radar echoes in the tesserae are not uniform with distance from Stuart; this lack of symmetry is consistent with the patchy nature of the parabolic deposits in the plains and the variability of large-scale structural patterns in Alpha that may affect the retention of fine debris on slopes.

We also examine two smaller craters, Peggy (11.9 km diameter) and Nadia (11 km diameter) that occur along the western margin of Alpha Regio (Fig. 3a). Nadia has a hummocky, radar-bright proximal ejecta blanket, but there are only traces of nearby lowerreturn areas in Magellan or Earth-based OC images. In the SC image (Fig. $3 \mathrm{~b}$ ), the entire southwestern margin of the plateau is darker than the north-central region of Alpha, but it is unlikely that this broad behavior is linked to Nadia deposits. The crater Peggy has a radar-dark surrounding deposit on the nearby tesserae in the $\mathrm{HH}$-polarization Magellan image, and this lower return (2.5 dB or $40 \%$ less than the adjacent terrain) is even more evident in the SC-polarization data (Fig. 4).

Also shown in Fig. 4 is an enigmatic low-return feature just northwest of Alpha Regio. This feature is generally consistent with an impact parabola, but the north-south distance between the
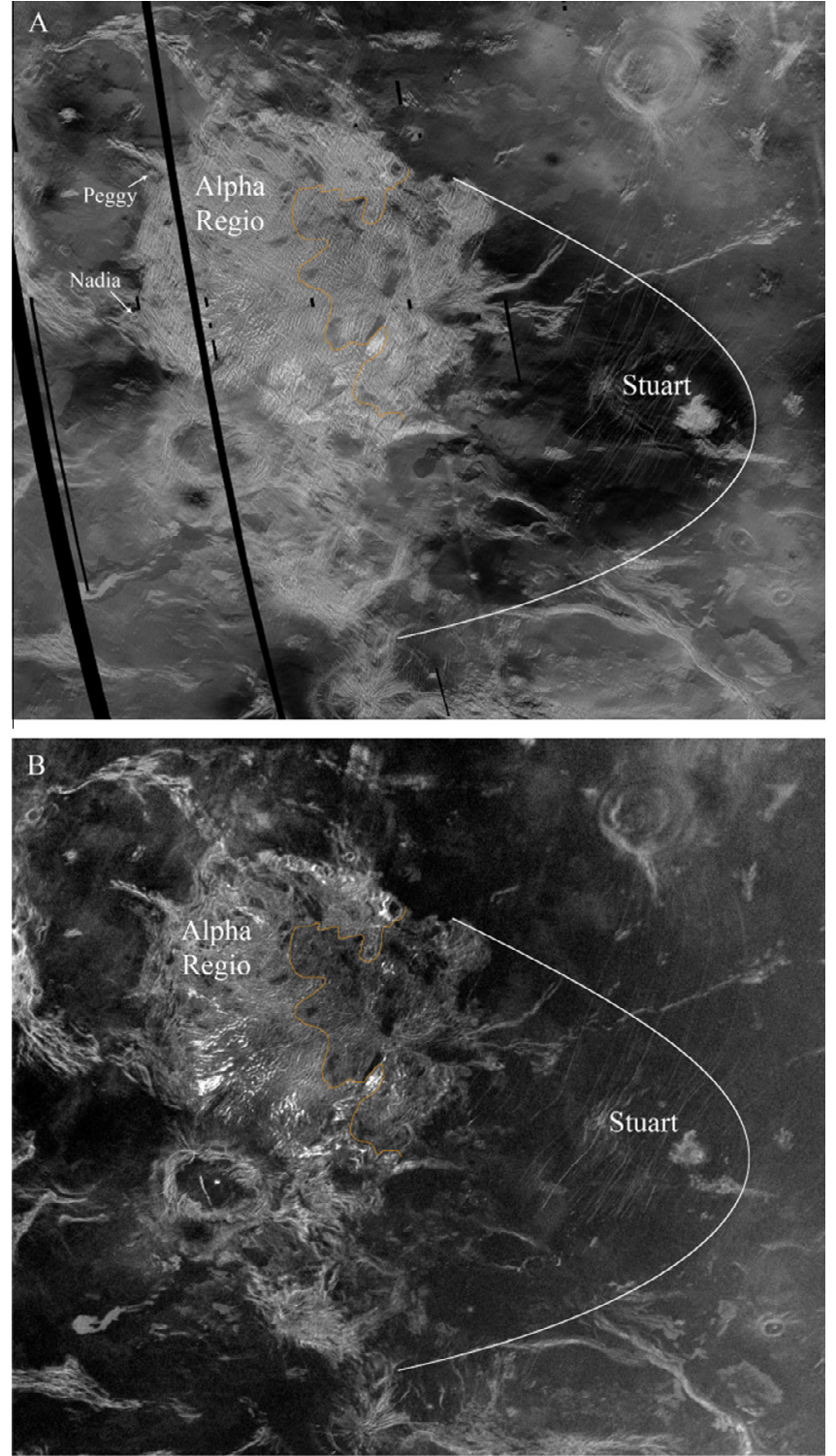

Fig. 3. Radar images of the region surrounding Alpha Regio (43.3 S to $13.3 \mathrm{~S}, 351.4 \mathrm{E}$ to $25.3 \mathrm{E}$ ); cylindrical projection. Image width about $3580 \mathrm{~km}$. (A) Magellan HHpolarization image. (B) Earth-based SC-polarization image. White curve shows bounds of parabola noted by Campbell et al. (1992). Orange outlines show approximate extent of region of decreased SC echoes, which we interpret to indicate fine-grained mantling of the highlands. (For interpretation of the references to color in this figure legend, the reader is referred to the web version of this article.)

wings is much smaller than expected. Even more intriguing is the lack of a clear crater near the eastern end of the deposit. There is a $6-\mathrm{km}$ diameter region of very modest $\mathrm{HH}$ echo enhancement centered between two small radar-dark portions of the wings, shown by the arrow in Fig. 4. We do not pursue the origin of the feature here, but it poses a challenge to models for the initial emplacement of parabolas and may indicate volcanic resurfacing of the parent crater.

How thick might the crater-derived debris on the tesserae be? The minimum depth of mantling material required to reduce the 12.6- $\mathrm{cm}$ wavelength echoes by some amount is derived from a study of the statistical roughness properties of terrestrial rockstrewn surfaces (Campbell, 2001). That work showed that the rms height at some length scale, and thus the radar backscatter coefficient in a polarization analogous to the SC return, varies with the parameters of an exponential rock size-frequency distribution, 


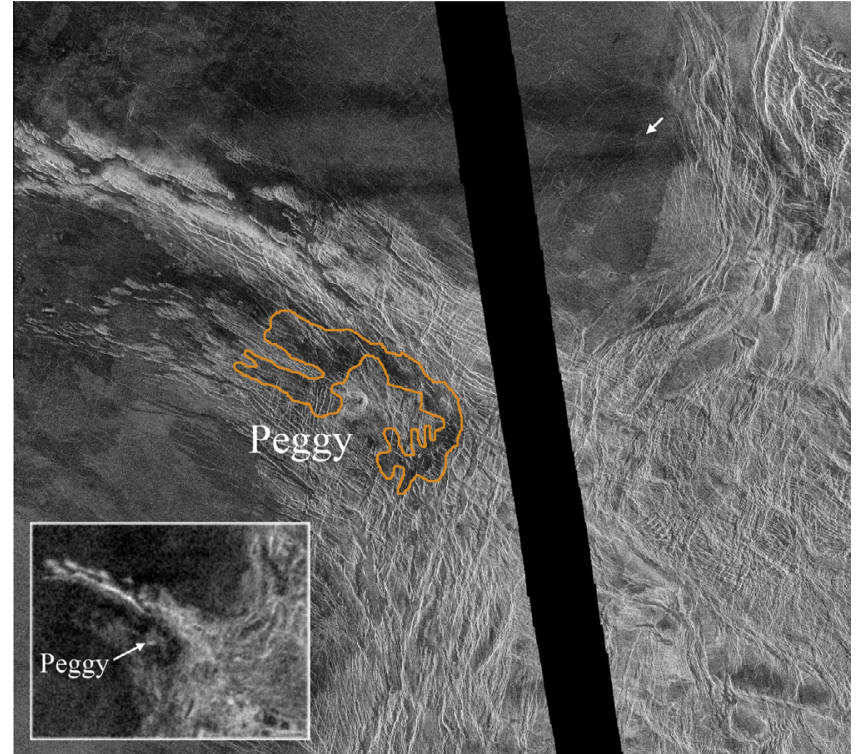

Fig. 4. Magellan HH-polarization radar image of the region surrounding Peggy crater $(11.9 \mathrm{~km}$ diameter) in northwestern Alpha Regio. Image width $450 \mathrm{~km}$. Orange outline shows the approximate bounds of low-return tessera terrain that we attribute to halo materials of Peggy. Inset figure shows Earth-based SC polarization data, which also show the low-return crater halo. White arrow denotes small feature at apparent source location of narrow parabolic feature. (For interpretation of the references to color in this figure legend, the reader is referred to the web version of this article.)

$N(d)=C e^{-\alpha d}$ where $C$ and $\alpha$ are scalar coefficients and $N$ is the number of rocks per square meter of surface per meter of diameter, $d$. By adding a flat interface of height $h$ above the surface that hosts the "perched" rocks, we calculated a revised value of the rms height in the presence of a smooth mantling layer.

Using a likely range of $\alpha$ from terrestrial field sites suggests that the $12.6-\mathrm{cm}$ wavelength radar echo declines by $0.2-0.3 \mathrm{~dB}$ per $\mathrm{cm}$ of mantle thickness as a function of the scaling term $C$. The change in echo is most dramatic for initially rock-poor terrain (low $C$ ), while rugged surfaces (high $C$ ) decline at the slower rate with $h$. A $25 \%$ brightness change thus implies $4-7 \mathrm{~cm}$ of smoothing material, and the $40 \%$ change near Peggy implies $8-12 \mathrm{~cm}$. It is important to note that these are minimum estimates, because we address only surface roughness and neglect the penetration of the radar signal through the mantling layer and scattering from the buried surface (e.g., Carter et al., 2004). If the dielectric loss of the debris is low, then we need a greater thickness to suppress the radar echo.

\subsection{Mukhina and Browning craters}

The region north of Gula Mons volcano hosts outcrops of tessera terrain that appear to be affected to varying degrees by extended crater deposits. Fig. 5a shows a Magellan view of the region, with the model-predicted parabolic deposit bounds of Mukhina (25 km diameter) and Browning (23.5 km diameter) craters plotted for reference. Both craters have at least a partial radar-dark, nearrim halo, but only Mukhina has clear streaks and patches of low radar return, up to $560 \mathrm{~km}$ from the crater, that suggest a moderately wind-eroded parabola. The rim and proximal ejecta of Mukhina have a higher overall radar brightness than that of Browning, consistent with the latter crater being older and more eroded. Whether other patches of lower radar return in the plains east of Mukhina are related to Browning is uncertain. Tesserae in the region comprise five small outcrops labeled $\mathrm{t} 1 \mathrm{-t} 5$ in Fig. 5a, and larger "blocks" forming Manzan-Gurme Tessera trend north from this area. All five smaller tessera outcrops have relatively high echo strength in the Magellan image, and high root-mean-square slope at the 10-100 m scale as inferred from Magellan altimeter observations (Ford and Pettengill, 1992).

As with the deposits of Stuart crater that appear to overlap Alpha Regio, the Earth-based SC-polarization image (Fig. 5b) provides insight into the possible distribution of crater debris in the Mukhina/Browning region. The average SC return over the outcrop farthest east of Mukhina, $t 5$, is about $1 \mathrm{~dB}(20 \%)$ brighter than similar echo averages over $\mathrm{t} 1$ or $\mathrm{t} 2$, and is comparable to the moderate-brightness portions of Manzan-Gurme Tessera to the north. If the general surface properties of $t 1$ through $t 5$ are similar, then this change in backscatter may indicate a spatially varying degree of mantling by debris from Mukhina. The fact that SC echoes are low immediately north (t2) and south (t 1$)$ of the crater is consistent with modeling by Vervack and Melosh (1992), which predicts that the thickest portion of a parabolic deposit occurs just east of a crater, with "wings" of deep material to the north and south of the central cavity. The lack of correlation between the SC backscatter and proximity to Browning crater may imply a minimal amount of original distal ejecta deposition, a relatively uniform contribution across the entire region, or a similar degree
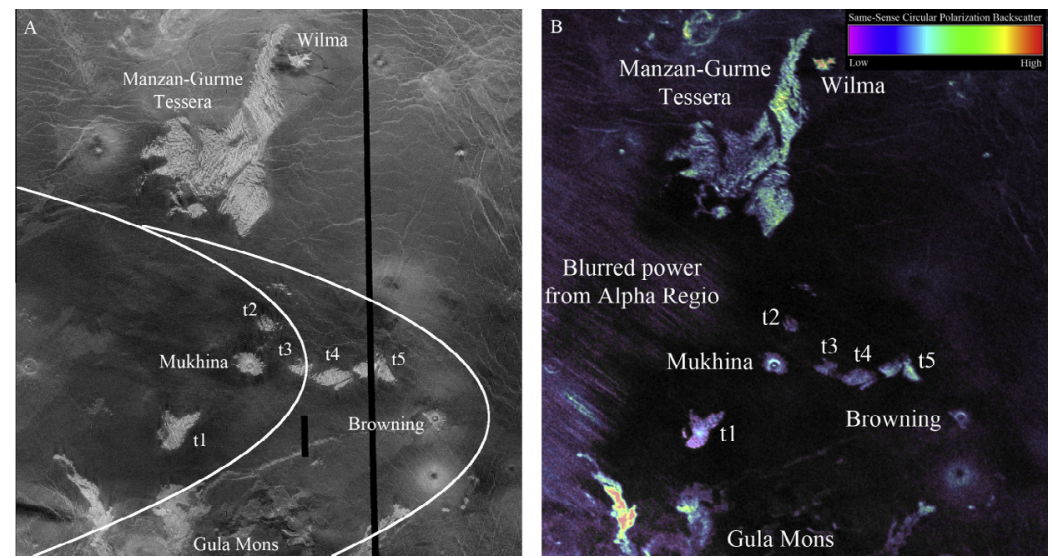

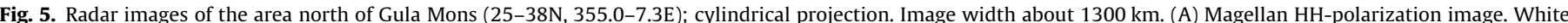

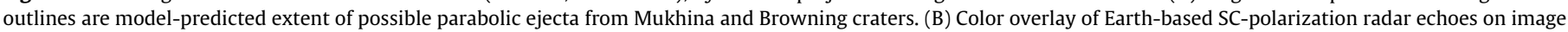

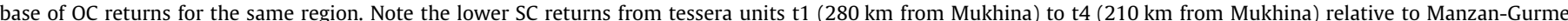

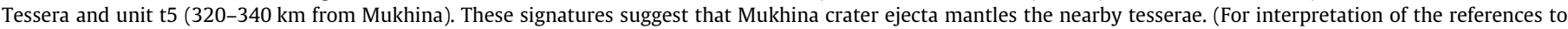
color in this figure legend, the reader is referred to the web version of this article.) 
of wind removal of its fine debris on the tesserae to that observed for the plains.

\subsection{Zirka Tessera}

Our third study region provides evidence that some crater debris in the tesserae may be more concentrated or longer-lived than deposits in nearby plains. We examine an area north and east of Beta Regio, where few-hundred km diameter tessera blocks form Hyndla Regio and the northeastern and southwestern parts of Zirka Tessera (Fig. 6). Three craters to the east of these tesserae could have emplaced fine-grained debris: 47-km diameter Patti, $28-\mathrm{km}$ West, and 18.7-km Bly. The 14-km diameter crater Wazata occurs along the margin of northeast Zirka Tessera (Basilevsky, 2008). Carter et al. (2006) note an enhanced linear-polarization signature from a plains area with numerous windstreaks between West crater and Hyndla Regio, suggesting a thin mantle of material that has been reworked by aeolian processes. None of the craters have clearly defined parabolic ejecta patterns in the Magellan radar image (Fig. 6a), but there is evidence for localized deposits of fine-grained mantling material.

In particular, there is a radar-dark patch in northeast Zirka Tessera, centered about $150 \mathrm{~km}$ southwest of Patti, that appears to be an extension of similar low-return deposits on the adjacent plains (Fig. 7). The radar-facing ridges of the tessera terrain are foreshortened and "lay over" toward the left in this image, and the backslopes of ridges are elongated. These backslope areas are much darker in the HH-polarization echoes than typical of the northeastern block of Zirka Tessera, and we infer that the surface is mantled to some thickness by extended ejecta of Patti. The degree of reduction in the radar return is larger than observed for the small outcrops around Mukhina crater, which retain a strong $\mathrm{HH}$-polarization echo (Fig. 5a). One possibility is that the mantling deposit in northeast Zirka Tessera is locally thicker, such that larger rocks are buried and small surface features are subdued. There are similar patches of low-return tessera terrain adjacent to the rugged proximal ejecta of Wazata crater (Fig. 8), consistent with the radar-dark haloes typical of many craters in the plains. This debris is distributed in an asymmetric pattern about the crater, due either to the initial ejecta emplacement pattern or subsequent erosion.

The detection of locally thick mantling material from the Magellan image is augmented by analysis of the Earth-based SC-polarization data (Fig. 6b). The radar-dark patch related to Patti crater (outlined on Fig. 6a) is quite evident, and is $2.8 \mathrm{~dB}$ (almost $50 \%$ ) lower in SC echo strength than typical areas of southwest Zirka Tessera. There are lower SC echoes in the tesserae concentric to this dark patch, making the remainder of northeastern Zirka 0.5-1.7 dB (10-30\%) darker than the southwestern block. Based on our analysis of mantling effects on 12.6-cm backscatter, the low-return patch is consistent with Patti ejecta at least $10-12 \mathrm{~cm}$ thick, with the rest of northeastern Zyrka mantled to at least $5-\mathrm{cm}$ average thickness. We cannot rule out the possibility that various blocks comprising Zirka Tessera have different initial surface properties, but the correlation of low-HH-return patches close to Patti and Wazata craters and lower SC returns in northeast Zirka Tessera suggests that more such material exists thinly across this entire block. A non-uniform deposition pattern of debris from Patti may have preferentially placed the fine-grained ejecta in/near the tesserae, but it is also possible that such materials have remained in the highlands for longer periods than debris in the plains.

\section{Discussion and implications for future exploration}

The Earth-based radar data allow mapping of widespread, crater-derived mantling deposits in the highlands that are not as evident in Magellan images. These deposits may be as thin as $5 \mathrm{~cm}$ in tesserae that retain a strong $\mathrm{HH}$-polarization backscatter intensity, but in some locales the ejecta could be $10-12 \mathrm{~cm}$ or greater in thickness. From these results we draw some basic conclusions.

A combination of emplacement conditions and subsequent erosion create non-uniform, potentially asymmetric surface coverage by fine-grained crater ejecta. There may thus be significant variability in the distal ejecta thickness and coverage pattern even among craters of similar diameter and age. The range of ejecta from the crater may be greater in some cases than predicted from a general model for parabola shape, as shown by our example for Stuart. Predictions of the global coverage by parabolic impact ejecta based on such models must thus be taken as conservative estimates - a given tessera site may experience a local enhancement of fine-grained material from a particular nearby crater. The rate of erosion and removal of ejecta in the tesserae is difficult
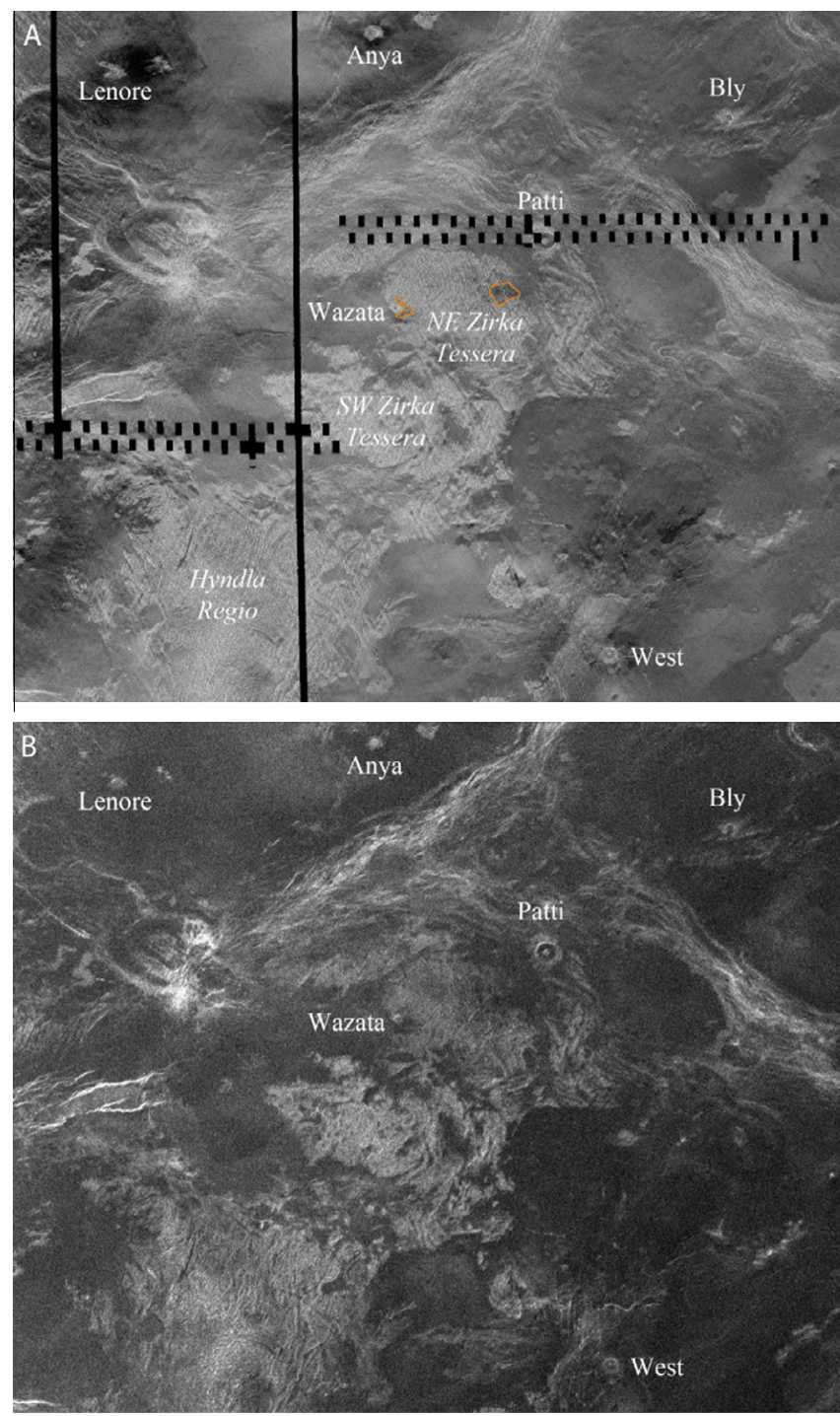

Fig. 6. (A) Magellan HH-polarization radar image of the region of Hyndla Regio and Zirka Tessera (25-40N, 290-308E). Image width about $1900 \mathrm{~km}$. Note that four craters (Wazata, Bly, Patti, and West) might contribute distal ejecta to the tesserae in this region. Orange outlines denote radar-dark deposits highlighted in Figs. 7 and 8. (B) Earth-based SC-polarization radar image of the same region. Note the lower overall backscatter of the northeastern part of Zirka Tessera relative to the highland terrain to the southwest. (For interpretation of the references to color in this figure legend, the reader is referred to the web version of this article.) 


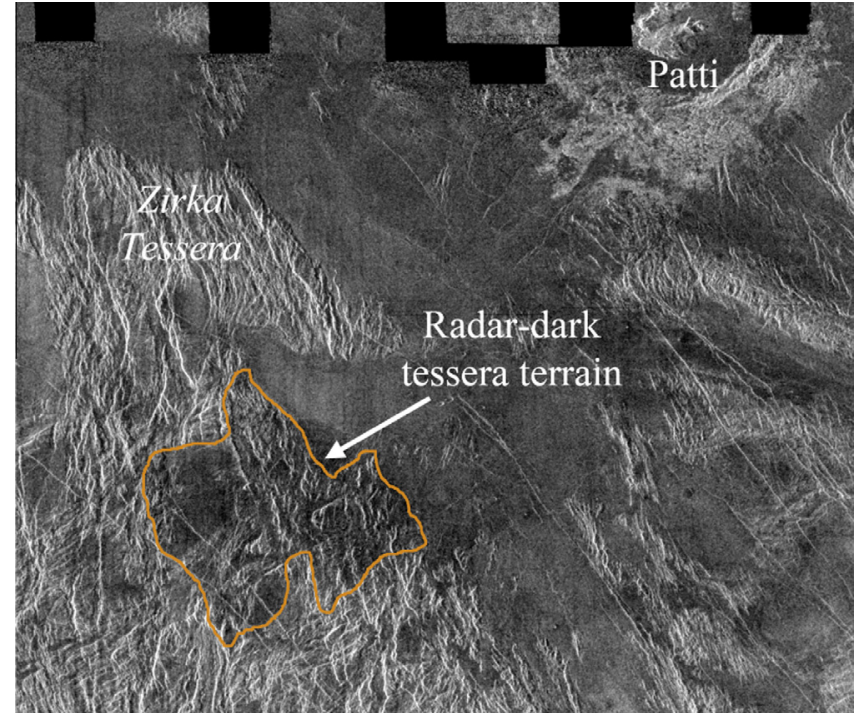

Fig. 7. Magellan HH-polarization, 75-m spatial resolution radar image of the region southwest of the 47-km diameter crater Patti. Image width about $203 \mathrm{~km}$. Orange outline highlights region of northeastern Zirka Tessera with much lower radar backscatter intensity, which we attribute to mantling by distal ejecta from Patti. (For interpretation of the references to color in this figure legend, the reader is referred to the web version of this article.)

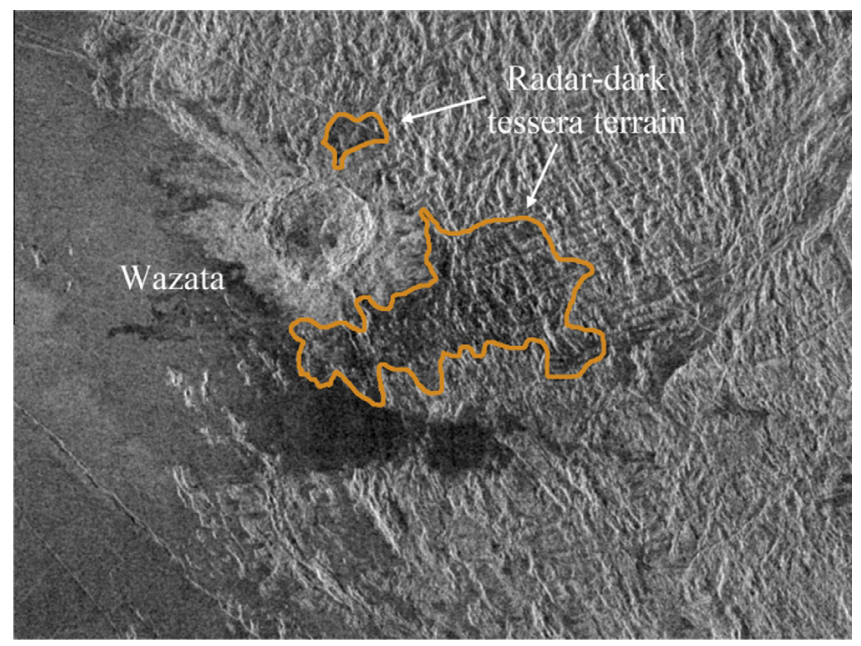

Fig. 8. Magellan HH-polarization, 75-m spatial resolution radar image of the region surrounding the 18-km diameter crater Wazata. Image width about $127 \mathrm{~km}$. Orange outline highlights region of Zirka Tessera with much lower radar backscatter intensity, which we attribute to mantling by ejecta from Wazata. (For interpretation of the references to color in this figure legend, the reader is referred to the web version of this article.)

to quantify, in part due to the patchy nature of the original depositional pattern. Surface wind speeds are greater at higher elevations, but the larger altimetric variability of the tesserae may create traps for debris that remain over longer timescales than in the plains.

Based on these factors, we suggest that future tessera landing sites, and locations for detailed interpretation of orbital infrared emissivity data, should be characterized by radar polarimetry as presented here. The major concern is "masking" of the tessera surface composition by ejecta from basaltic plains regions (e.g., Basilevsky et al., 2004), but even craters in the tesserae may create debris that is significantly altered from the original target material. Similar concerns arise for regions at elevations (about $6053 \mathrm{~km}$ planetary radius for equatorial sites, increasing to $6055 \mathrm{~km}$ at high latitudes) where high-dielectric precipitates are known to occur from anomalously low microwave emissivity values (Pettengill et al., 1992; Arvidson et al., 1994; Campbell et al., 1999). Requiring polarimetric radar coverage, at present, limits the geographic range to those regions visible from Earth near inferior conjunction.

The potentially large spatial extent of thin ejecta mantling on the Venus highlands also motivates a new look at Magellan observations despite the geometric complications associated with the radar polarization. Of great interest is to establish the relative timescales of crater-debris removal from the plains and tesserae, and to investigate whether some highland areas might preserve ejecta from craters now partly or fully buried by the volcanic plains. Future studies of the tesserae would benefit from additional Earth-based or orbital radar imaging, improved topographic maps, and pathfinding photography by descent probes or aero-vehicles.

\section{Acknowledgments}

Helpful manuscript reviews provided by M. Gilmore and an anonymous referee are most appreciated. The authors gratefully acknowledge support from the staff of the Arecibo Observatory and the Green Bank Telescope in collecting the Venus radar maps. This work was funded in part by NASA Planetary Mission Data Analysis Program (PMDAP) Grant NNX13AL17G, and by NASA Grant NNX10AP64G, “Arecibo Radar Observations of NEO's and Other Solar System Bodies". The Arecibo Observatory is operated by SRI International under a cooperative agreement with the National Science Foundation (AST-1100968), and in alliance with Ana G. Méndez-Universidad Metropolitana, and the Universities Space Research Association. The National Radio Astronomy Observatory is a facility of the National Science Foundation operated under cooperative agreement by Associated Universities, Inc. As part of the PMDAP-supported work, backscatter images from the 1988 and 2012 Venus observations are being prepared for submission to the NASA Planetary Data System.

\section{References}

Arvidson, R.E., Brackett, R., Shepard, M.K., Izenberg, N.R., Fegley, B., Plaut, J.., 1994 Microwave signatures and surface properties of Ovda Regio and surroundings. Icarus $112,171-186$.

Barsukov, V.L. et al., 1986. The geology and geomorphology of the Venus surface as revealed by the radar images obtained by Veneras 15 and 16. J. Geophys. Res. 91 (suppl.), D378-D398 (Proc. Lun. Plan. Sci. Conf. 16th).

Basilevsky, A.T., 2008. Geologic Map of the Beta Regio Quadrangle (V-17), Venus. USGS Scientific Investigations Map 3023.

Basilevsky, A.T., Head, J.W., Abdrakhimov, A.M., 2004. Impacr crater air fall deposits on the surface of Venus: Areal distribution, estimated thickness, recognition in surface panoramas, and implications for provenance of sampled surface materials. J. Geophys. Res. 109. http://dx.doi.org/10.1029/2004JE002307.

Basilevsky, A.T., Ivanov, M.A., Head, J.W., Aittola, M., Raitala, J., 2007. Landing on Venus: Past and future. Planet. Space Sci. 55, 2097-2112.

Basilevsky, A.T. et al., 2012. Geologic interpretation of the near-infrared images of the surface taken by the Venus Monitoring Camera, Venus Express. Icarus 217, 434-450.

Bender, K.C., Williams, K.K., Homan, K.S., 2000. Tectonic evaluation of Alpha Regio highland and environs, Venus. Icarus 148, 153-159.

Bindschadler, D.L., Head, J.W., 1988. Diffuse scattering of radar on the surface of Venus: Origin and implications for the distribution of soils. Earth, Moon Planets 42, 133-149.

Bindschadler, D.L., Head, J.W., 1989. Characterization of Venera 15/16 geologic units from Pioneer Venus reflectivity and roughness data. Icarus 77, 3-20.

Bindschedler, D.L., DeCharon, A., Beratan, K.K., Smrekaer, S.E., Head, J.W., 1992 Magellan observations of Alpha Regio: Implications for formation of complex ridged terrains on Venus. J. Geophys. Res. 97, 13563-13577. http://dx.doi.org/ 10.1029/92JE01332.

Bondarenko, N., Head, JW. 2009. Crater-associated dark diffuse features on Venus: Properties of surficial deposits and their evolution. J. Geophys. Res. 114, E03004.

Campbell, B.A., 2001. Radar backscatter from Mars: Properties of rock-strewn surfaces. Icarus $150,38-47$

Campbell, B.A., Campbell, D.B., 1992. Analysis of volcanic surface morphology on Venus from comparison of Arecibo, Magellan, and terrestrial airborne radar data. J. Geophys. Res. 97, 16293-16314. 
Campbell, B.A., Shepard, M.K., 1996. Lava flow surface roughness and depolarized radar scattering. J. Geophys. Res. 101, 18941-18952.

Campbell, D.B. et al., 1989. Styles of volcanism on Venus: New Arecibo high resolution radar data. Science 246, 373-377.

Campbell, D.B et al., 1992. Magellan observations of extended impact crater related features on the surface of Venus. J. Geophys. Res. 97 (E10), 16249-16277.

Campbell, B.A., Campbell, D.B., DeVries, C.H., 1999. Surface processes in the Venus highlands: Results from analysis of Magellan and Arecibo data. J. Geophys. Res, 104 (E1), 1897-1916.

Campbell, B.A. et al., 2007. Focused 70-cm radar mapping of the Moon. IEEE Trans. on Geoscience and Remote Sensing 45 (12), 4032-4042. http://dx.doi.org/ 10.1109/TGRS.2007.906582.

Campbell, B.A., Carter, L.M., Hawke, B.R., Campbell, D.B., Ghent, R.R., 2008. Volcanic and impact deposits of the Moon's Aristarchus Plateau: A new view from Earthbased radar images. Geology 36, 135-138.

Carter, L.M., Campbell, D.B., Campbell, B.A., 2004. Impact crater related surficial deposits on Venus: Multi-polarization radar observations with Arecibo. J. Geophys. Res. 109, E06009. http://dx.doi.org/10.1029/2003JE002227.

Carter, L.M., Campbell, D.B., Campbell, B.A., 2006. Volcanic deposits in shield fields and highland regions on Venus: Surface properties from radar polarimetry. J. Geophys. Res. 111. http://dx.doi.org/10.1029/2005JE002519.

Chadwick, D.J., Schaber, G.G., 1993. Impact crater outflows on Venus: Morphology and emplacement mechanisms. J. Geophys. Res. 98, 20891-20902.

Ford, P.G., Pettengill, G.H., 1992. Venus topography and kilometer-scale slopes. J. Geophys. Res. 97, 13102-13114.

Garvin, J.B., 1990. The global budget of impact-derived sediments on Venus. Earth, Moon Planets 50-51, 175-190.

Ghent, R.R., Leverington, D.W., Campbell, B.A., Hawke, B.R., Campbell, D.B., 2005. Earth-based observations of radar-dark crater haloes on the Moon: Implications for regolith properties. J. Geophys. Res. 110 (E2), E02005. http://dx.doi.org/ 10.1029/2004JE002366.

Gilmore, M.S., Head, J.W., 2000. Sequential deformation of plains at the margins of Alpha Regio, Venus: Implications for tesserae formation. Meteor. Planet. Sci. 35, 667-687.
Hagfors, T., 1964. Backscattering from an undulating surface with applications to radar returns from the Moon. J. Geophys. Res. 69, 3779-3784.

Hansen, V.L., Banks, B.K., Ghent, R.R., 1999. Tessera terrain and crustal plateaus, Venus. Geology 27, 1071-1074.

Hashimoto, G.L. et al., 2008. Felsic highland crust on Venus suggested by Galileo near-infrared mapping spectrometer data. J. Geophys. Res. 113, E00B24.

Helbert, J. et al., 2008. Surface brightness variations seen by VIRTIS on Venus Express and implications for the evolution of the Lada Terra region, Venus. Geophys. Res. Lett. 35, L11201.

Herrick, R.R., Phillips, R.J., 1994. Implications of a global survey of venusian impact craters. Icarus 111, 387-416.

Ivanov, M.A., Head, J.W., 1996. Tessera terrain on Venus: A survey of the globa distribution, characteristics, and relation to surrounding units from Magellan data. J. Geophys. Res. 101 (E6), 14861-14908.

Ivanov, M.A., Head, J.W., 2011. Global geologic map of Venus. Planet. Space Sci. 59 1559-1600.

Izenberg, N.R., Arvidson, R.E., Phillips, R.J., 1994. Impact crater degradation on the Venus plains. Geophys. Res. Lett. 21, 289-292.

McKinnon, W.B., Zahnle, KJ. Ivanov, B.A., Melosh, H.J., 1997. Cratering on Venus: Models and observations. In: Bougher, S.W., Hunten, D.M., Phillips, R.J. (Eds.) Venus II Geology, Geophysics, Atmosphere, and Solar Wind Environment. University of Arizona Press, Tucson, pp. 969-1014.

Pettengill, G.H., Ford, P.G., Wilt, R.J., 1992. Venus surface radiothermal properties as observed by Magellan. J. Geophys. Res. 97, 13091-13102.

Report of the Venus Science and Technology Definition Team, 2009. Venus Flagship Mission Study, JPL.

Schaller, C.J., Melosh, H.J., 1998. Venusian ejecta parabolas: Comparing theory with observations. Icarus 131, 123-137. http://dx.doi.org/10.106/icar.1997.5855.

Vervack, R.J., Melosh, H.J., 1992. Wind interaction with falling ejecta: Origin of the parabolic features on Venus. Geophys. Res. Lett. 19, 525-528. 